\title{
You Only Group Once: Efficient Point-Cloud Processing with Token Representation and Relation Inference Module
}

\author{
Chenfeng Xu* ${ }^{1}$, Bohan Zhai* ${ }^{1}$, Bichen $\mathrm{Wu}^{*}{ }^{2}$, Tian $\mathrm{Li}^{3}$, Wei Zhan ${ }^{1}$ \\ Peter Vajda ${ }^{2}$, Kurt Keutzer ${ }^{1}$ and Masayoshi Tomizuka ${ }^{1}$
}

\begin{abstract}
D point-cloud-based perception is a challenging but crucial computer vision task. A point-cloud consists of a sparse, unstructured, and unordered set of points. To understand a point-cloud, previous point-based methods, such as PointNet++, extract visual features through hierarchically aggregation of local features. However, such methods have several critical limitations: 1) Such methods require several sampling and grouping operations, which slow down the inference speed. 2) Such methods spend an equal amount of computation on each points in a point-cloud, though many of points are redundant. 3) Such methods aggregate local features together through downsampling, which leads to information loss and hurts the perception performance. To overcome these challenges, we propose a novel, simple, and elegant deep learning model called YOGO (You Only Group Once). YOGO divides a point-cloud into a small number of parts and extracts a high-dimensional token to represent points within each subregion. Next, we use self-attention to capture token-to-token relations, and project the token features back to the point features. We formulate the mentioned series of operation as a relation inference module (RIM). Compared with previous methods, YOGO only needs to sample and group a point-cloud once, so it is very efficient. Instead of operating on points, YOGO operates on a small number of tokens, each of which summarizes the point features in a sub-region. This allows us to avoid computing on the redundant points and thus boosts efficiency. Moreover, YOGO preserves point-wise features by projecting token features to point features although the computation is performed on tokens. This avoids information loss and can improve point-wise perception performance. We conduct thorough experiments to demonstrate that $Y O G O$ achieves at least 3.0x speedup over point-based baselines while delivering competitive classification and segmentation performance on the ShapeNetParts and S3DIS datasets. The code is available at https://github.com/chenfengxu714/YOGO.git
\end{abstract}

\section{INTRODUCTION}

Increasing applications, such as autonomous driving, robotics, augmented and virtual reality, require efficient and accurate 3D perception. As LiDAR and other depth sensors become popular, 3D visual information is commonly captured, stored, and represented as point-clouds. A point-cloud is essentially a set of unstructured points. Each point in a pointcloud consists of its 3D-coordinates and, optionally, features like normal, color, and intensity.

Different from images, where pixels are arranged as 2D grids, points in a point-cloud are unstructured. This makes

\footnotetext{
* denotes equal contribution.

${ }^{1}$ Chenfeng Xu, Bohan Zhai, Wei Zhan, Kurt Keutzer and Masayoshi Tomizuka are with University of California, Berkeley. Correspondence to xuchenfeng@berkeley.edu

${ }^{2}$ Bichen Wu and Peter Vajda are with Facebook Reality Labs.

3 Tian Li is with Peking University.
}

it infeasible to aggregate features by using convolutions, the de facto operator for image-based vision models. In order to handle the unstructured point-cloud, popular methods such as PointNet [1] process each point through multi-layer perceptrons and use a global pooling to aggregate point-wise features to form a global feature. Then, the global feature is shared with all the point-wise features. This mechanism allows points within a point-cloud to communicate local features with each other. However, such pooling mechanism is too coarse as it fails to aggregate and process local information in a hierarchical way.

Another mainstream approach, PointNet++ [2], extends PointNet [1] by hierarchically organizing point-cloud. Precisely, they sample center points, group nearby neighbours, and apply PointNet to aggregate local features. After PointNet++, hierarchical feature extraction [3]-[5] has been further developed, but several limitations are not adequately addressed: 1) Sampling and grouping for point-cloud are handcraft operations, and hierarchically operating them is computationally heavy. 2) This feature extraction requires computation on all points, many of which are redundant because adjacent points don't provide extra information. 3) As local features are aggregated, point-wise features are discarded, which leads to information loss.

Based on the aforementioned motivation, instead of directly max-pooling the whole point-cloud into one single feature (PointNet [1]) or traversing to aggregating the neighbors for many points (PointNet++ [2]), we propose a better way to aggregate point-cloud features, as shown in Fig 1 We only need to group points into a few sub-regions once, which has less computation cost than previous point-based methods. Then each sub-region is squeezed into a token representation [6], which concisely summarizes the pointwise features within a region and eliminates redundancy. Next, we apply self-attention [7] to capture the relations between each token (region). While the representations are computed on the token-level, we also use a cross-attention module to project computed token features to point-wise features, which preserves full details with minimum computational cost. The series of operations above is what we call relation inference modules (RIM), and we propose YOGO which is composed of a stack of relation inference modules.

Our proposed YOGO can efficiently and effectively handle 3D point-cloud object classification, part segmentation, and scene segmentation. For the object classification task, YOGO receives the raw point sets with $\mathrm{x}, \mathrm{y}, \mathrm{z}$ coordinates and will output the class based on the tokens in the final RIM layer. 


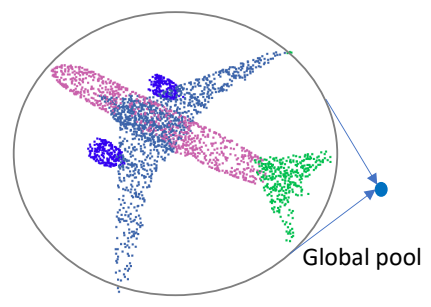

(a) PointNet

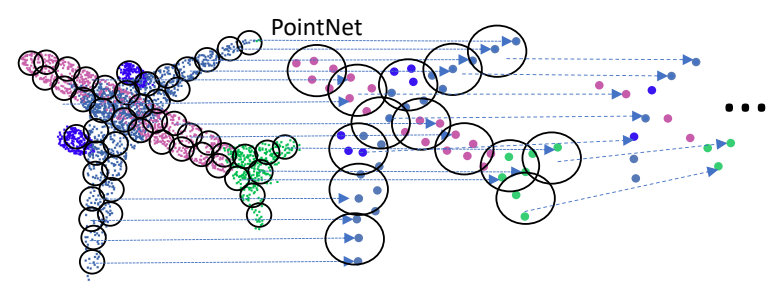

(b) PointNet++

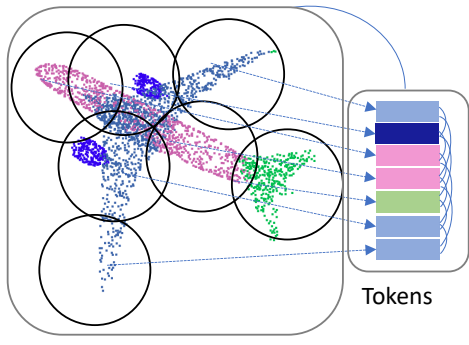

(c) YOGO

Fig. 1: (a) PointNet [1] directly globally pools a point-cloud into a global feature. (b) PointNet++ [2] hierarchically samples amounts of points, groups their nearby points, and aggregates the nearby points into one point via pointNet. (c) YOGO divides the whole point-cloud into several parts, squeezes it into several tokens and models the token-token relations to capture the part structure of the car and the token-points relations to project the tokens into the original points.

For the segmentation task, YOGO also leverages the original points and outputs the per-point labels based on the final point-features. We conduct experiments on ShapeNetParts and S3DIS datasets respectively, to demonstrate the efficiency and efficacy of the proposed method. Specifically, $Y O G O$ outperforms and achieves 3.0x speedup over the classic PointNet++ [2]. We also provide extensive arguments for how the model works to obtain such improvements in the experiment section. Note that we do not expect to beat all the state-of-the-art methods via superior local-feature aggregation operators since they have evolved over several years. Instead, we provide a new, simple, and elegant baseline from the perspective of relational inference. We do expect it can inspire the research community to explore this approach further.

Overall, the key contributions of our work are as follows:

- We design a new, simple and elegant baseline termed $Y O G O$ which efficiently and effectively processes the unordered point-cloud.

- We provide thorough empirical analysis on the efficacy and efficiency of our method.

- We illustrate the relations extracted via the proposed $Y O G O$ and develop intuitive explanations for its performance.

\section{RELATED WORK}

\section{A. Point-Cloud Processing}

Recent point-cloud processing methods mainly include volumetric-based method, projection-based method, and pointbased method. We briefly introduce them as follows.

Volumetric-based method is to rasterize the point-cloud into 3D grids (voxels) [8]-[11] thus the convolution operator can be easily applied. However, the number of voxels is usually limited to no more than $3^{3}=27$ or $5^{5}=125$ due to the constraint of GPU memory [11]. Thereby many points are grouped into the same voxel, which introduces information loss. Besides, To structure a regular format, many empty voxels are added for padding, which causes redundant computation. Using a permutohedral lattice reduces the kernel to 15 lattices [12], yet the numbers are still limited. Recently, sparse convolutions [13] are proposed to apply on the nonempty voxels [14]-[17], largely improving the efficiency of
3D convolutions and boosting the scales of voxel numbers and the models.

Projection-base method attempts to project the 3D pointcloud into a 2D plane and exploit 2D convolution to extract features [18]-[24]. Specifically, the bird-eye-view projection [25], [26] and the spherical projection [19]-[21], [27] make great progresses in outdoor point-cloud segmentation (e.g. autonomous driving). However, it is hard for them to capture the point-cloud geometry structure especially when the pointcloud becomes complex, such as indoor scenes [28] and object parsing [29] since the points are collected in an irregular manner.

Point-based method is to directly process the point-cloud. The most classic method PointNet [1] consumes the points by MLP network and squeezes the final high-dimensional features into a single global feature via global pooling, which significantly improves the performance of the pointcloud recognition and semantic segmentation. Furthermore, PointNet++ [2] extends it into a hierarchical form, in each layer the local features are aggregated. Many works further develop advanced local-feature aggregation operators that mimics the convolution with customised method to structure data [3], [4], [30]-[34]. However, the unstructured format of the point-cloud dramatically hinders their efficiency since many point-based methods rely on hierarchical handcraft operations like farthest point sampling, $\mathrm{K}$ nearest neighbors and ball query. Although they gradually downsample the points in each layer, the sample-group operations are required to be applied in each stage, which makes it inefficient.

Our proposed $Y O G O$ is a new pipeline regarding the pointbased method, which only needs to sample-group the points once and is robust to the various sample-group operations.

\section{B. Self-attention for perception}

Self-attention was first introduced by Vaswani et al. [7] for the natural language processing and recently becomes especially popular in the perception field [6], [35]-[38]. It has great advantages to model the relations among per-elements such as tokens (for sequence data [7], [39]) and pixels (for image data [35]) without complex data rearrangement. This is very useful for point-cloud data because it avoids structuring 


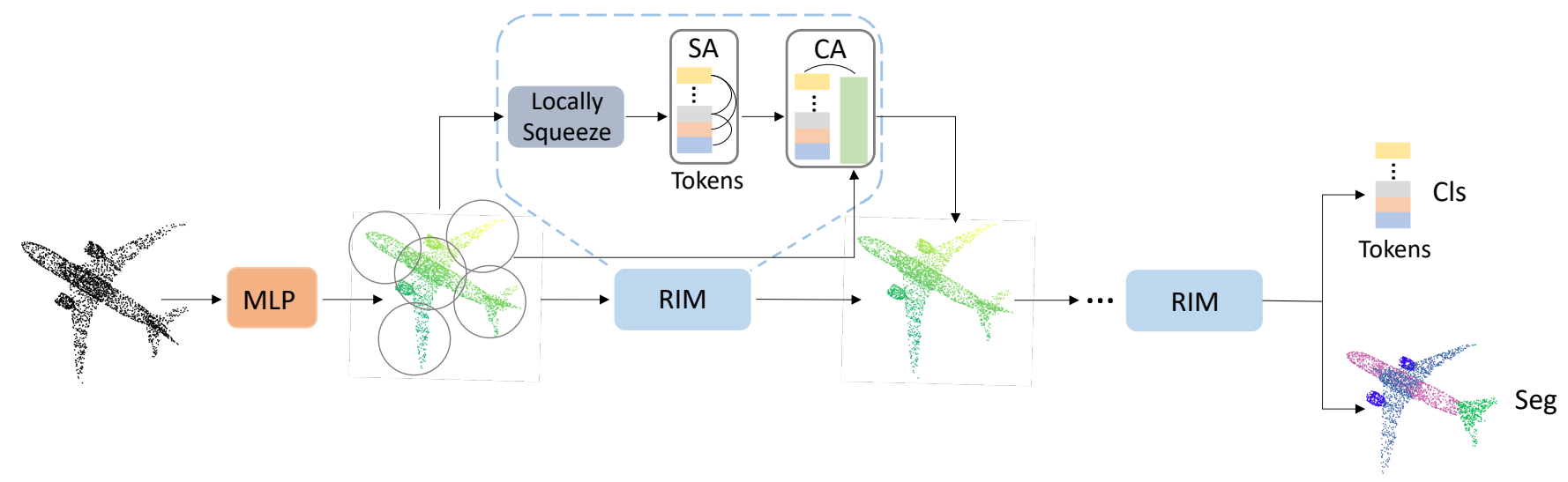

Fig. 2: The framework of YOGO. It consists of several stacked relation inference module (RIM). "Locally Squeeze" indicates that we locally squeeze the features into several tokens based on the sub-regions, "SA" means self-attention module and "CA" means cross-attention module.

the data as previous point-based method did. Yet, few works explore self-attention on point-cloud, a main challenge is that the self-attention operation is computationally intensive with the time complexity of $O\left(N^{2}\right)$ and makes the model unscalable. For commonly used point-cloud dataset [28], [29], [40], at least 1024 points are required to recognize the pointcloud, which is a relatively large number for self-attention. Thus, this motivates us to explore how to efficiently take advantage of the self-attention mechanism.

In this paper, different from the previous point-based methods that aim to design a more advanced local-feature aggregation operator, we propose a new baseline model termed $Y O G O$ that takes advantage of the pooling operation and the self-attention. In particular, instead of directly applying the self-attention to process the point-cloud, we use pooling operation to aggregate the features (in a subregion) so that we only preserve important features and can apply the self-attention in an efficient manner to model the relations between the important features. The details will be introduced in the method section.

\section{METHOD}

\section{A. Overview}

We propose $Y O G O$, a deep learning model for point-cloud classification and segmentation. YOGO accepts a set of points as input $\left\{\mathbf{p}_{i}\right\}$, each point $\mathbf{p}_{i}=\left(x_{i}, y_{i}, z_{i}\right)$ is parameterized by its $3 \mathrm{D}$ coordinates and optionally, additional features such as normal, color, and so on. At the output, for classification, YOGO generates a global prediction $\mathbf{y}$, and for semantic segmentation, it generates point-wise labels $\left\{\mathbf{y}_{i}\right\}$.

$Y O G O$ consists of a stack of relation inference modules (RIM), which is an efficient module to process a point-cloud. For a point-cloud as input $\left\{\mathbf{p}_{i}\right\}$ with $N$ points, we first divide it to $L$ sub-regions by uniform sampling $L$ centers and grouping their neighboring points. For points within each sub-region, we use pooling operations to extract a few tokens representing this region. The tokens are then processed by self-attention to capture the feature interactions within each region. Next, tokens are processed by a cross-attention module to exchange

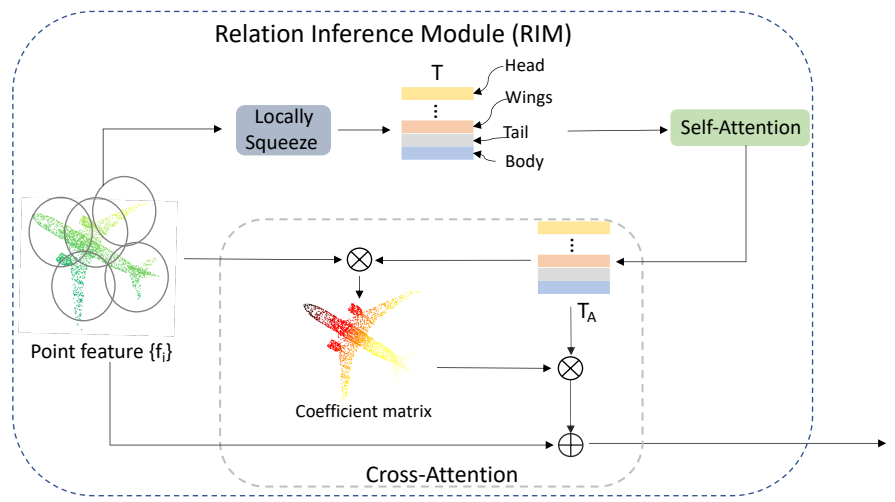

Fig. 3: Relation Inference Module (RIM). The input point features $\left\{\mathbf{f}_{\mathbf{i}}\right\}$ are first locally squeezed into several tokens, then we can get a stronger token representation $\mathbf{T}_{\mathbf{A}}$ via selfattention module. Next, the tokens $\mathbf{T}_{\mathbf{A}}$ are projected into the original point-wise features in the cross-attention module. The coefficient matrix shown in the figure is a sample from a token.

information among regions and aggregate long-range features. After this, we project the token features back to point-wise features, to obtain a stronger point-wise representation.

\section{B. Sub-region Division}

We divide the point-cloud into $L(L \ll N)$ regions via the commonly used farthest point sampling (FPS) and $K$ nearest neighbouring search (KNN search) or ball query [2]. Different from the previous point-based methods [2], [3] that re-sample and re-group the points in each layer, we only need to sample-group once because the grouped indices can be re-used for gathering nearby points in each layer. Also note that the point sampling is fast since $L \ll N$ and $L$ is same in each layer. After this step, the point-cloud $\left\{\mathbf{p}_{i}\right\}$ is divided into $L$ sub-regions $\left\{\mathbf{p}_{i}^{(j)}\right\}$ where $\mathbf{p}_{i}^{(j)}$ denotes the $i^{t h}$ point in the $j^{\text {th }}$ sub-region. 


\section{Relation Inference Module (RIM)}

The relation inference module (RIM) does not require handcrafted grouping and sampling operations to aggregate point-wise features. Instead, it adopts a simple pooing operation an self-attention. The pooling operation is used to aggregate features within a sub-region [1], [31], while self-attention is used to capture relations between sub-regions. Self-attention has been demonstrated to have a superior ability to capture long-range relations in many vision and language tasks. However, as self-attention's computational cost is quadratic with number of input elements, it is infeasible to be directly used for point-cloud. In our work, our design is to let self-attention operate on region/tokens, instead of points, this greatly reduces the computational cost while leveraging the advantages of self-attention. The structure of RIM is presented in Fig. 3

Specifically, given a set of point features $\left\{\mathbf{f}_{i}\right\}$ corresponding to a point-cloud $\left\{\mathbf{p}_{i}\right\}$, we divide the points into sub regions $\left\{\mathbf{p}_{i}^{(j)}\right\}$ with corresponding features $\left\{\mathbf{f}_{i}^{(j)}\right\}$. RIM computes a token to represent points within each region as

$$
\mathbf{T}^{(j)}=G\left(\operatorname{maxpool}_{\mathbf{i}}\left(\left\{\mathbf{f}_{\mathbf{i}}^{(\mathbf{j})}\right\}\right)\right),
$$

where $\mathbf{f}_{i}^{(j)} \in \mathbb{R}^{1 \times C}$ denotes point- $i$ in region- $j$. $\operatorname{maxpool}_{i}$ is the max-pooling operation to squeeze points in $\left\{\mathbf{f}_{i}^{(j)}\right\}$ to one. $G(\cdot)$ is a linear function that maps the pooled feature to the output $\mathbf{T}^{(j)} \in \mathbb{R}^{1 \times C_{T}}$. This is similar to PointNet [1], yet PointNet applies a pooling to all the points, while we focus on different sub-regions.

Next, to model the relations between different regions using self-attention as [7]. We first combine all the tokens $\left\{\mathbf{T}^{(j)}\right\}$ to form a tensor $\mathbf{T} \in \mathbb{R}^{L \times C_{T}}$. Since self-attention is a permutation-invariant operation, we can choose any order to stack $\left\{\mathbf{T}^{(j)}\right\}$ to form $\mathbf{T}$. On the token, we compute

$$
\begin{gathered}
M_{t t}=\mathbf{T} \hat{W}_{k}\left(\mathbf{T} \hat{W}_{q}\right)^{\mathrm{T}} \\
S A(\mathbf{T})=\operatorname{softmax}\left(M_{t t}\right)\left(T \hat{W}_{v}\right)+\mathbf{T}, \\
\mathbf{T}_{\mathbf{A}}=S A(\mathbf{T}) \hat{W}_{p} .
\end{gathered}
$$

where $\mathbf{T}_{\mathbf{A}} \in \mathbb{R}^{L \times C_{T}}$ is the output of the self-attention module, $M_{t t} \in \mathbb{R}^{L \times L}$ is the coefficient matrix regarding token-token relation in $\mathbf{T} \in \mathbb{R}^{L \times C_{T}}$, and $\hat{W}_{p} \in \mathbb{R}^{C_{T} \times C_{T}}, \hat{W}_{v} \in \mathbb{R}^{C_{T} \times C_{T}}$, $\hat{W}_{k} \in \mathbb{R}^{C_{T} \times C_{T}}, \hat{W}_{q} \in \mathbb{R}^{C_{T} \times C_{T}}$ are the parameterized matrix, representing the weights for project, value, key and query [7], respectively.

Next, we project the output tokens back to point-wise features. To do this, we feed $T_{A} \in \mathbb{R}^{L \times C_{T}}$ and the original point-wise feature $\left\{\mathbf{f}_{i}\right\}$ to a cross-attention module as

$$
\begin{gathered}
\overline{\mathbf{T}}_{\mathbf{A}}=G^{\prime}\left(\mathbf{T}_{\mathbf{A}}\right), \\
M_{t p}=\mathbf{f}_{i} W_{k}\left(\overline{\mathbf{T}}_{\mathbf{A}} W_{q}\right)^{\mathrm{T}}, \\
C A\left(\overline{\mathbf{T}}_{\mathbf{A}}, \mathbf{f}_{i}\right)=\operatorname{softmax}\left(M_{t p}\right)\left(\overline{\mathbf{T}}_{\mathbf{A}} W_{v}\right)+\mathbf{f}_{i}, \\
\overline{\mathbf{f}}_{i}=C A\left(\overline{\mathbf{T}}_{\mathbf{A}}, \mathbf{f}_{i}\right) W_{p},
\end{gathered}
$$

where $\overline{\mathbf{f}}_{i}$ is the output point-feature of the cross-attention module, which is parametrized by the matrix $W_{p} \in \mathbb{R}^{C \times C}$, the matrix $W_{v} \in \mathbb{R}^{C \times C}$, the matrix $W_{k} \in \mathbb{R}^{C \times C}$, and the matrix
$W_{q} \in \mathbb{R}^{C \times C} . G^{\prime}$ is a MLP mapping the $\mathbf{T}_{\mathbf{A}} \in \mathbb{R}^{L \times C_{T}}$ into $\overline{\mathbf{T}}_{\mathbf{A}} \in \mathbb{R}^{L \times C}$ and $M_{t p} \in \mathbb{R}^{L \times 1}$ is a coefficient matrix between the tokens $\overline{\mathbf{T}}_{\mathbf{A}}$ and a point feature $\mathbf{f}_{i}$.

Analytically, each token element in $\mathbf{T}_{\mathbf{A}}$ encodes the most important and representative components in each corresponding sub-region. The self-attention regarding the $\mathbf{T}_{\mathbf{A}}$ can help capture the structure of the point-cloud, e.g., as shown in Fig. 3. the head, the wings, the airplane body, and the tail have strong structure relations between each other. This is useful to learn semantics since we can recognize the airplane through these representative elements. After this, the coefficient matrix $M_{t p}$ is calculated to indicate the relations between the tokens and each point which helps to project tokens with rich semantic information into original point-cloud features.

\section{Training objective}

Our proposed network $Y O G O$ can handle point-cloud classification and segmentation tasks and can be simply trained end-to-end. The tokens and the point feature in the final $R I M$ are used for classification and segmentation tasks, respectively. We simply apply the commonly used crossentropy loss to train the model for both classification and segmentation task, which are respectively given by

$$
L_{c l s}=-\sum_{c=1}^{C} \hat{y_{c}} \cdot \log \left(p_{c}\right)
$$

where $L_{c l s}$ is the cross-entropy loss for classification, $C$ is the number of categories of a point-cloud, $\hat{y_{c}}$ is the ground truth and $p_{c}$ is the softmax probability prediction to the $c^{t h}$ class, then

$$
L_{s e g}=\frac{-\sum_{N} \sum_{c=1}^{C} y_{n_{c}} \cdot \log \left(p_{n_{c}}\right)}{N},
$$

where $L_{\text {seg }}$ is for segmentation, $C$ is the total categories of each point, $N$ is the whole number of the point-cloud, $y_{n_{c}}$ ) is the ground-truth for $n^{\text {th }}$ point, and $p_{n_{c}}$ is the softmax probability prediction.

\section{EXPERIMENT}

The experiments are conducted on the ShapeNetParts [29] and S3DIS [28] for segmentation task. We conduct both comparison experiments for effectiveness and efficiency evaluation and ablation study for deep analysis of why $Y O G O$ works. The details are illustrated as follows.

\section{A. Implementation detail}

We stack eight relation inference modules on all experiments and set $C_{T}$ equal to 256 and $C$ equal to 32, 32, 64, $64,128,128,256,256$ respectively for the eight relation inference modules.

We first conduct the experiment on the ShapeNetParts [29] dataset, which involves 16681 different objects with 16 categories and 2-6 part labels for each. By utilizing the evaluation metric mean intersection-over-union (mIoU), we first calculate the part-averaged IoU for each of the 2874 test models and average the values as the final metrics. We set the $L$ equal to $32, K$ equal to 96 . All the coordinates 


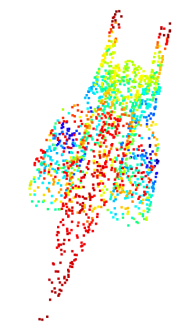

Token 3

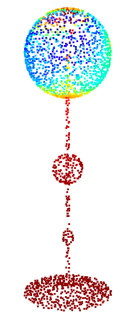

Token 1

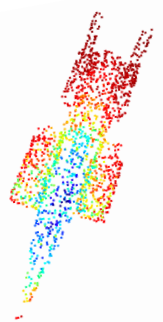

Token 7

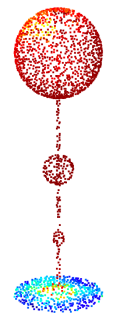

Token 4

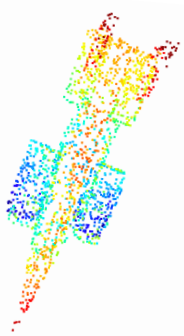

Token 11

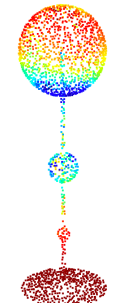

Token 11

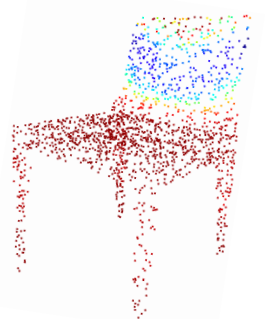

Token 2

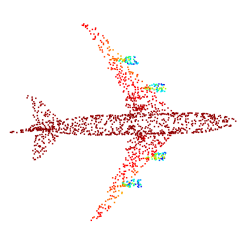

Token 1

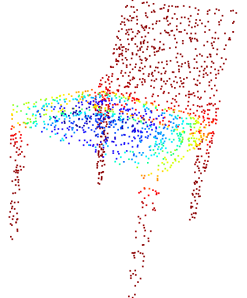

Token 11

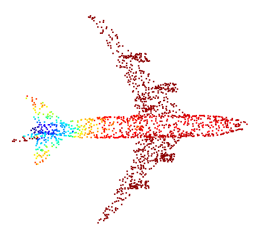

Token 8

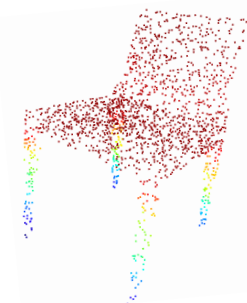

Token 14

Fig. 4: Visualization of the coefficient matrix in cross-attention module on the ShapeNetParts dataset. Blue means the response is small and Red means the response is large. Taking the rocket point-cloud and the chair point-cloud as examples, as for the rocket point-cloud, we choose token 3, token 7, and token 11 that attend the head, the tail, and the tail, respectively. As for the chair point-cloud, we choose token 2, token 11, and token 14 that attend the sitting board and the legs, the back board and the legs, the back board and the stting board, respectively.

of points are normalized into $[0,1]$. As for the ball query grouping, we set the radium to 0.2 . During training, we input the data with the size of 2048 points and the batch size of 128 , and use the cosineAnnealing learning rate strategy with initial learning rate 1e-3 and Adam optimizer to optimize the network. During the inference, we input the points with the size of 2048 and vote 10 times for each point-cloud.

We then conduct the experiment on the S3DIS [28], which is collected from the real-world indoor scenes and includes 3D scans of Matterport Scanners from 6 areas. We also use the mIoU as the evaluation metric. We apply the 1, 2, 3, 4, 6 areas for training and use the 5 area for the test since it is the only area that does not overlap with any other area. We set the $L$ equal to $32, K$ equal to 128 . For ball query grouping, the radium is set to 0.3 . We train the networks via feeding the input with the 4096 points and batch size 64, and utilize the same training strategy as the aforementioned for ShapeNetParts dataset. During the inference, we also input the points with the size of 4096 and vote one times for each point-cloud.

Finally, we apply our method to the classification tasks on the ModelNet40 dataset [40]. This is a CAD dataset with 12311 meshed models and contains 40 categories. We set $L$ equal to $16, K$ equal to 128 . For ball query grouping, the radium is set to 0.15 . We train the network with the input of 1024 points and batch size 64, and use the same training strategy as [3]. Note that all the experiments are conducted on one Titan RTX GPU.

\section{B. Comparison experiment}

We first perform YOGO on the ShapeNetParts and S3DIS datasets for semantic segmentation evaluation, as shown in Table. I and Table. II] We can observe that YOGO has very competitive performance and speedups at least $3 \mathrm{x}$ over point-based baselines on the ShapeNetParts dataset. Precisely, YOGO slightly outperforms the classic baseline PointNet++ [2] and performs at least 3x faster. Although the unofficial PointNet++ [41] slightly outperforms YOGO, YOGO largely speedups it over 9.2x faster. As for the S3DIS dataset, YOGO also achieves at least 4.0x speedup and delivers competitive performance. PointCNN [3] outperforms $Y O G O$ over 0.9 mIoU (resp. $3.26 \mathrm{mIoU}$ ) on the ShapeNetParts (resp. S3DIS) dataset but is not efficient. Regarding the classification task conducted on the ModelNet40 dataset presented as Table. III. YOGO still has competitive performance over the popular baselines.

We can also observe that $Y O G O$ with two different grouping method KNN and ball query have similar performance, which shows that our proposed method is relatively stable with regard to different grouping strategies. Note that there remain many excellent methods that perform better than YOGO, we are not attempting to beat all of them. Instead, we propose a novel baseline from the perspective of token-token relation and token-point relation.

\section{Ablation study}

In this subsection, we do an in-depth study of our proposed $Y O G O$, including the effectiveness of the Relation Inference Module (RIM) and related techniques on the ShapeNetParts 


\begin{tabular}{cccc}
\hline Method & Mean IoU & Latency & GPU Memory \\
\hline PointNet [1] & 83.7 & $21.4 \mathrm{~ms}$ & $1.5 \mathrm{~GB}$ \\
RSNet [42] & 84.9 & $73.8 \mathrm{~ms}$ & $0.8 \mathrm{~GB}$ \\
SynSpecCNN [43] & 84.7 & - & - \\
PointNet++ [2] & 85.1 & $77.7 \mathrm{~ms}$ & $2.0 \mathrm{~GB}$ \\
PointNet++* [2] & 85.4 & $236.7 \mathrm{~ms}$ & $0.9 \mathrm{~GB}$ \\
DGCNN [44] & 85.1 & $86.7 \mathrm{~ms}$ & $2.4 \mathrm{~GB}$ \\
SpiderCNN [45] & 85.3 & $170.1 \mathrm{~ms}$ & $6.5 \mathrm{~GB}$ \\
SPLATNet [12] & 85.4 & - & - \\
SO-Net [33] & 84.9 & - & - \\
PointCNN [3] & 86.1 & $134.2 \mathrm{~ms}$ & $2.5 \mathrm{~GB}$ \\
\hline YOGO (KNN) & 85.2 & $25.6 \mathrm{~ms}$ & $0.9 \mathrm{~GB}$ \\
YOGO (Ball query) & 85.1 & $21.3 \mathrm{~ms}$ & $1.0 \mathrm{~GB}$ \\
\hline
\end{tabular}

TABLE I: Quantitative results of semantic segmentation on the ShapeNetPart dataset. The latency and GPU memory are measured under the batch size of 8 and the point number of 2048. PointNet++* is a reproduced version from the popular repository [41].

\begin{tabular}{cccc}
\hline Method & Mean IoU & Latency & GPU Memory \\
\hline PointNet [1] & 42.97 & $24.8 \mathrm{~ms}$ & $1.0 \mathrm{~GB}$ \\
DGCNN [44] & 47.94 & $174.3 \mathrm{~ms}$ & $2.4 \mathrm{~GB}$ \\
RSNet [42] & 51.93 & $111.5 \mathrm{~ms}$ & $1.1 \mathrm{~GB}$ \\
PointNet++* [2] & 50.7 & $501.5 \mathrm{~ms}$ & $1.6 \mathrm{~GB}$ \\
TangentConv [46] & 52.6 & - & - \\
PointCNN [3] & 57.26 & $282.43 \mathrm{~ms}$ & $4.6 \mathrm{~GB}$ \\
\hline$Y O G O$ (KNN) & 54.0 & $27.7 \mathrm{~ms}$ & $2.0 \mathrm{~GB}$ \\
$Y O G O$ (Ball query) & 53.8 & $24.0 \mathrm{~ms}$ & $2.0 \mathrm{~GB}$ \\
\hline
\end{tabular}

TABLE II: Quantitative results of semantic segmentation on the S3DIS dataset. The latency and GPU memory are measured under the batch size of 8 , the point number of 4096. PointNet++* is a reproduced version from the popular repository [41].

\begin{tabular}{cc}
\hline Method & Overall Accuracy \\
\hline PointNet [1] & 89.2 \\
PointNet++ [2] & 90.7 \\
SO-Net [33] & 90.9 \\
SpiderCNN [45] & 90.5 \\
MCConv [47] & 90.9 \\
PointCNN [3] & 92.2 \\
DGCNN [44] & 92.2 \\
ViT-B-2* [36] & 78.9 \\
Transformer* [7] & 82.1 \\
Perceiver [48] & 85.7 \\
\hline YOGO (KNN) & 91.4 \\
YOGO (Ball query) & 91.3 \\
\hline
\end{tabular}

TABLE III: Quantitative results on the ModelNet40 dataset. The results of ViT-B-2* and Transformer* are directly taken from Perceiver [48].

Parts dataset. More importantly, we analyze what RIM learns, thus explicitly explain why $Y O G O$ works.

The effectiveness of RIM. The self-attention module and the cross-attention module are the two key components in RIM. We study them by removing them respectively. In particular, when we remove the self-attention module (SA), we insert several MLP layers that have similar FLOPs as the self-attention modules. When we remove the cross-attention module, we use different related techniques: 1) Directly squeeze the tokens $T_{A}$ into one global feature and concatenate it with the point feature $F_{P}$, which is similar to PointNet [1]; 2) add or concatenate the tokens $T_{A}$ into the $F_{P}$ corresponding to different sub-regions. The results are shown in Table. IV]

We can observe that if we coarsely use the tokens by squeezing them into one global feature, the performance is largely worse than the original $Y O G O$ by $1.1 \mathrm{mIoU}$. A more reasonable method is to leverage them on the corresponding sub-regions by adding/concatenating each token with the corresponding point features of sub-regions. It can be seen that the original $Y O G O$ still outperforms them by at least $0.5 \mathrm{mIoU}$, which shows the cross-attention method is more effective. On the other hand, when we substitute the selfattention module with several MLP, the performance also drops $0.5 \mathrm{mIoU}$, which also shows that it is important to learn the relations between different sub-regions.

Visualization analysis of RIM. To deeper explore what RIM learns, we visualize some samples of the coefficient matrix in the cross-attention module, as shown in Fig. 4. Note that the coefficient matrix of the cross-attention module indicates the relation between the tokens and the original point features, yet there is no explicit supervision to guide the learning coefficient matrix. We can observe that different tokens can automatically attend to different parts even without supervision. For example, the tokens regarding the rocket point-cloud have stronger responses to the head, the tail, and the flank, respectively. Similarly, the tokens regarding the chair point-cloud have stronger responses to the back, the sitting board, and the legs, respectively. These can demonstrate that tokens indeed carry strong semantic information, and the cross-attention module indeed helps the tokens with rich semantic information be projected into point-wise features.

We also visualize the coefficient matrix in the self-attention module, for better visualization, we choose the models with $L$ equal to 16. As shown in Fig. 5, the figure (a) in the right shows the token attending the body has more relations to the token indicating the flank and the wings, which together form a whole airplane. The figure (c) and (d) on the right are also similar, they attend different sub-regions which are connected together and build the structure of the airplane point-cloud. We find that for the tokens attending the relatively same parts, their coefficient values are low, which indicates that the self-attention module does not simply learn the similarity of different tokens instead capture the relations of different sub-regions that build the structure of a point-cloud. This can also be indicated from the small coefficient values between the same tokens.

Other techniques. We study other techniques related to our proposed $Y O G O$ including the effectiveness of choices of $L$ and $K$, the sampling and grouping methods, and the different pooling strategies.

Regarding the choices of $L$ and $K$, we conduct experiments by fixing the $K$ equal to 64 and increasing the $L$ under both farthest point sampling (FPS) and random sampling, as well as fixing the $L$ equal to 32 and increasing the $K$ under both KNN and ball query [2], as presented in the Fig. 6. We find that when using different sampling methods, the performance 


\begin{tabular}{cc}
\hline Method & Mean IoU \\
\hline$Y O G O$ w/o CA (concat global feature) & 84.1 \\
$Y O G O$ w/o CA (adding tokens) & 84.7 \\
$Y O G O$ w/o CA (concat tokens) & 84.6 \\
$Y O G O$ w/o SA & 84.7 \\
\hline$Y O G O$ & 85.2 \\
\hline
\end{tabular}

TABLE IV: Ablation study for self-attention module and cross-attention module in RIM. YOGO represents that we are using the full modules, YOGO w/o SA means no self-attention module, YOGO w/o CA means no cross-attention.

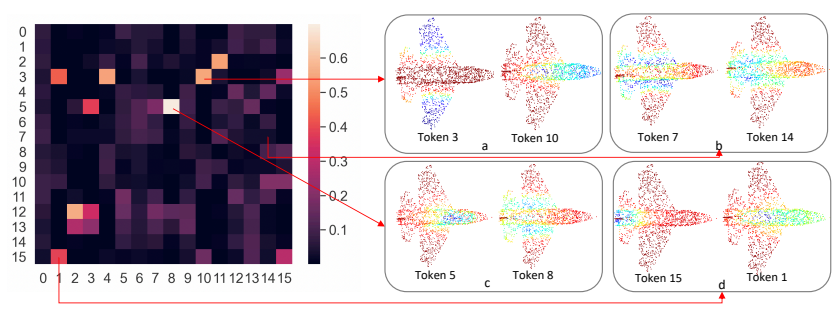

Fig. 5: Visualization of the coefficient matrix (left) in selfattention module and the corresponding attentions to different parts (right). Blue (resp. red) means the response is small (resp. large). The figure (a), (b), (c), (d) show the token 3 (resp. token 10) attending the body (resp. the wings and tail), the token 7 (resp. token 14) attending the wings and head (resp. the wings and head), the token 5 (resp. token 8) attending the wings and tail (resp. the wings and head), the token 15 (resp. token 1) and the wings (resp. head to the wings and tail), respectively. We can observe that the token 3 and the token 10 in figure (a), and token 5 and token 8 in figure (c) correspond to large coefficient value since their attending parts have strong structure relation, which can form a airplane. Yet token 7 and token 14 in figure (b) correspond to small coefficient value since they attend very similar part thus have less structure relation.

will be better if increasing $L$. Besides, even for random sampling strategy, it has relatively small margin to farthest point sampling, which may indicate that our proposed YOGO is robust to different sampling methods. When using different grouping method, the performance also consistently improves when $K$ become larger. Besides, we find there is not too much performance gap between ball query and KNN, this indicate our method is robust to different grouping methods.

Regarding the choices of different pooling operations, we study the differences between average pooling and max pooling, as shown in Table. $\mathrm{V}$ The experiment demonstrates the max pooling achieves higher performance than the average pooling. The reason is that the max pooling can better aggregate the most important information in a sub-region, which is more important for our proposed YOGO since it highly depends on their relations.

\section{DISCUSSION AND CONCLUSION}

In this paper, we propose a novel, simple, and elegant framework YOGO (you only group once) for efficient point-cloud processing. Different from previous point-based methods,

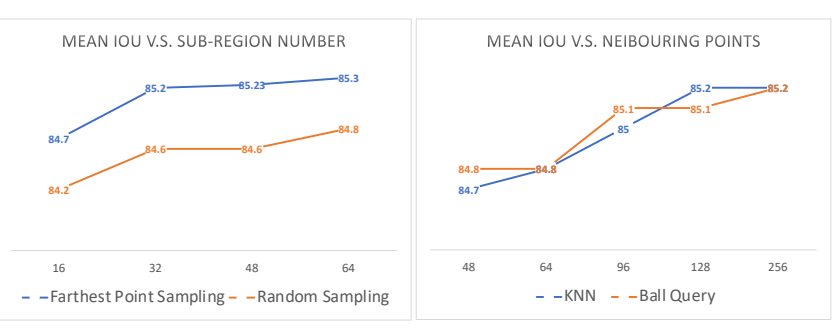

Fig. 6: The left figure shows the effect of the number of sub-regions regarding the farthest point sampling and random sampling and the right figure shows the effect of the number of grouping points regarding the $\mathrm{KNN}$ grouping and ball query grouping.

\begin{tabular}{cc}
\hline Method & Mean IoU \\
\hline$Y O G O$ with max-pool & 85.2 \\
YOGO with avg-pool & 85.0 \\
\hline
\end{tabular}

TABLE V: Ablation study for different pooling strategies.

YOGO divides point-cloud into a few sub-regions applying sampling and grouping operation once, leverages efficient token representation, models token relations and projects token to obtain strong point-wise features. YOGO achieves at least $3 \mathrm{x}$ speedup over popular point-based baselines. Meanwhile, YOGO delivers competitive performance on semantic segmentation on ShapeNetParts and S3DIS dataset, and classification on ModelNet40 dataset. Moreover, YOGO is also robust to different sampling and grouping strategies, even for random sampling. It is noteworthy to mention that $Y O G O$ introduced in this paper is a new framework and has huge potentials for point-cloud processing. In particular, it can be improved from the aspects of how to better sample then group out sub-regions, how to obtain better tokens, and how to better combine tokens and point-wise features etc. We hope that it can inspire the research community to further develop this framework.

\section{REFERENCES}

[1] C. R. Qi, H. Su, K. Mo, and L. J. Guibas, "Pointnet: Deep learning on point sets for 3d classification and segmentation," in CVPR, 2017.

[2] C. R. Qi, L. Yi, H. Su, and L. J. Guibas, "Pointnet++: Deep hierarchical feature learning on point sets in a metric space," in NIPS, 2017.

[3] Y. Li, R. Bu, M. Sun, W. Wu, X. Di, and B. Chen, "Pointenn: Convolution on $\chi$-transformed points," in Proceedings of the 32nd International Conference on Neural Information Processing Systems, 2018, pp. 828-838.

[4] Y. Liu, B. Fan, G. Meng, J. Lu, S. Xiang, and C. Pan, "Densepoint: Learning densely contextual representation for efficient point cloud processing," in Proceedings of the IEEE/CVF International Conference on Computer Vision, 2019, pp. 5239-5248.

[5] H. Thomas, C. R. Qi, J.-E. Deschaud, B. Marcotegui, F. Goulette, and L. J. Guibas, "Kpconv: Flexible and deformable convolution for point clouds," Proceedings of the IEEE International Conference on Computer Vision, 2019.

[6] B. Wu, C. Xu, X. Dai, A. Wan, P. Zhang, M. Tomizuka, K. Keutzer, and P. Vajda, "Visual transformers: Token-based image representation and processing for computer vision," arXiv preprint arXiv:2006.03677, 2020.

[7] A. Vaswani, N. Shazeer, N. Parmar, J. Uszkoreit, L. Jones, A. N. Gomez, L. Kaiser, and I. Polosukhin, "Attention is all you need," 2017. 
[8] D. Maturana and S. Scherer, "Voxnet: A 3d convolutional neural network for real-time object recognition," in 2015 IEEE/RSJ International Conference on Intelligent Robots and Systems (IROS). IEEE, 2015, pp. 922-928.

[9] Y. Ben-Shabat, M. Lindenbaum, and A. Fischer, "3dmfv: Threedimensional point cloud classification in real-time using convolutional neural networks," IEEE Robotics and Automation Letters, vol. 3, no. 4, pp. 3145-3152, 2018.

[10] X. Roynard, J.-E. Deschaud, and F. Goulette, "Classification of point cloud scenes with multiscale voxel deep network," arXiv preprint arXiv: 1804.03583, 2018.

[11] Z. Liu, H. Tang, Y. Lin, and S. Han, "Point-voxel cnn for efficient 3d deep learning," arXiv preprint arXiv:1907.03739, 2019.

[12] H. Su, V. Jampani, D. Sun, S. Maji, E. Kalogerakis, M.-H. Yang, and J. Kautz, "Splatnet: Sparse lattice networks for point cloud processing," in Proceedings of the IEEE conference on computer vision and pattern recognition, 2018, pp. 2530-2539.

[13] B. Liu, M. Wang, H. Foroosh, M. Tappen, and M. Pensky, "Sparse convolutional neural networks," in Proceedings of the IEEE conference on computer vision and pattern recognition, 2015, pp. 806-814.

[14] C. Choy, J. Gwak, and S. Savarese, "4d spatio-temporal convnets: Minkowski convolutional neural networks," in Proceedings of the IEEE/CVF Conference on Computer Vision and Pattern Recognition, 2019, pp. 3075-3084.

[15] H. Tang, Z. Liu, S. Zhao, Y. Lin, J. Lin, H. Wang, and S. Han, "Searching efficient $3 \mathrm{~d}$ architectures with sparse point-voxel convolution," in European Conference on Computer Vision. Springer, 2020, pp. 685702.

[16] H. Zhou, X. Zhu, X. Song, Y. Ma, Z. Wang, H. Li, and D. Lin, "Cylinder3d: An effective 3d framework for driving-scene lidar semantic segmentation," arXiv preprint arXiv:2008.01550, 2020.

[17] Y. Yan, Y. Mao, and B. Li, "Second: Sparsely embedded convolutional detection," Sensors, vol. 18, no. 10, p. 3337, 2018.

[18] Z. Wang, W. Zhan, and M. Tomizuka, "Fusing bird's eye view lidar point cloud and front view camera image for $3 \mathrm{~d}$ object detection," in 2018 IEEE Intelligent Vehicles Symposium (IV). IEEE, 2018, pp. 1-6.

[19] B. Wu, A. Wan, X. Yue, and K. Keutzer, "Squeezeseg: Convolutional neural nets with recurrent crf for real-time road-object segmentation from 3d lidar point cloud," in ICRA, 2018.

[20] B. Wu, X. Zhou, S. Zhao, X. Yue, and K. Keutzer, "Squeezesegv2: Improved model structure and unsupervised domain adaptation for road-object segmentation from a lidar point cloud," in ICRA, 2019.

[21] C. Xu, B. Wu, Z. Wang, W. Zhan, P. Vajda, K. Keutzer, and M. Tomizuka, "Squeezesegv3: Spatially-adaptive convolution for efficient point-cloud segmentation," in European Conference on Computer Vision. Springer, 2020, pp. 1-19.

[22] H. Su, S. Maji, E. Kalogerakis, and E. Learned-Miller, "Multi-view convolutional neural networks for $3 \mathrm{~d}$ shape recognition," in Proceedings of the IEEE international conference on computer vision, 2015, pp. 945-953.

[23] F. J. Lawin, M. Danelljan, P. Tosteberg, G. Bhat, F. S. Khan, and M. Felsberg, "Deep projective 3d semantic segmentation," in International Conference on Computer Analysis of Images and Patterns. Springer, 2017, pp. 95-107.

[24] A. Boulch, B. Le Saux, and N. Audebert, "Unstructured point cloud semantic labeling using deep segmentation networks." $3 D O R$, vol. 2, p. 7, 2017.

[25] B. Yang, W. Luo, and R. Urtasun, "Pixor: Real-time 3d object detection from point clouds," in Proceedings of the IEEE conference on Computer Vision and Pattern Recognition, 2018, pp. 7652-7660.

[26] A. H. Lang, S. Vora, H. Caesar, L. Zhou, J. Yang, and O. Beijbom, "Pointpillars: Fast encoders for object detection from point clouds," in Proceedings of the IEEE/CVF Conference on Computer Vision and Pattern Recognition, 2019, pp. 12697-12 705.

[27] A. Milioto, I. Vizzo, J. Behley, and C. Stachniss, "Rangenet++: Fast and accurate lidar semantic segmentation," in 2019 IEEE/RSJ International Conference on Intelligent Robots and Systems (IROS). IEEE, 2019, pp. 4213-4220.

[28] I. Armeni, S. Sax, A. R. Zamir, and S. Savarese, "Joint 2d-3d-semantic data for indoor scene understanding," arXiv preprint arXiv:1702.01105, 2017.

[29] A. X. Chang, T. Funkhouser, L. Guibas, P. Hanrahan, Q. Huang, Z. Li, S. Savarese, M. Savva, S. Song, H. Su et al., "Shapenet: An informationrich 3d model repository," arXiv preprint arXiv:1512.03012, 2015.
[30] B.-S. Hua, M.-K. Tran, and S.-K. Yeung, "Pointwise convolutional neural networks," in Proceedings of the IEEE Conference on Computer Vision and Pattern Recognition, 2018, pp. 984-993.

[31] Z. Liu, H. Hu, Y. Cao, Z. Zhang, and X. Tong, "A closer look at local aggregation operators in point cloud analysis," in European Conference on Computer Vision. Springer, 2020, pp. 326-342.

[32] P.-S. Wang, Y. Liu, Y.-X. Guo, C.-Y. Sun, and X. Tong, "O-cnn: Octree-based convolutional neural networks for 3d shape analysis," ACM Transactions on Graphics (TOG), vol. 36, no. 4, pp. 1-11, 2017.

[33] J. Li, B. M. Chen, and G. H. Lee, "So-net: Self-organizing network for point cloud analysis," in Proceedings of the IEEE conference on computer vision and pattern recognition, 2018, pp. 9397-9406.

[34] A. Komarichev, Z. Zhong, and J. Hua, "A-cnn: Annularly convolutional neural networks on point clouds," in Proceedings of the IEEE/CVF Conference on Computer Vision and Pattern Recognition, 2019, pp. $7421-7430$.

[35] N. Carion, F. Massa, G. Synnaeve, N. Usunier, A. Kirillov, and S. Zagoruyko, "End-to-end object detection with transformers," in European Conference on Computer Vision. Springer, 2020, pp. 213229.

[36] A. Dosovitskiy, L. Beyer, A. Kolesnikov, D. Weissenborn, X. Zhai, T. Unterthiner, M. Dehghani, M. Minderer, G. Heigold, S. Gelly et al., "An image is worth $16 \times 16$ words: Transformers for image recognition at scale," arXiv preprint arXiv:2010.11929, 2020.

[37] P. Sun, R. Zhang, Y. Jiang, T. Kong, C. Xu, W. Zhan, M. Tomizuka, L. Li, Z. Yuan, C. Wang, and P. Luo, "SparseR-CNN: End-to-end object detection with learnable proposals," arXiv preprint arXiv:2011.12450, 2020.

[38] Z. Zhu, M. Xu, S. Bai, T. Huang, and X. Bai, "Asymmetric non-local neural networks for semantic segmentation," in Proceedings of the IEEE/CVF International Conference on Computer Vision, 2019, pp. 593-602.

[39] J. Devlin, M.-W. Chang, K. Lee, and K. Toutanova, "Bert: Pre-training of deep bidirectional transformers for language understanding," arXiv preprint arXiv:1810.04805, 2018.

[40] P. Shilane, P. Min, M. Kazhdan, and T. Funkhouser, "The princeton shape benchmark," in Proceedings Shape Modeling Applications, 2004. IEEE, 2004, pp. 167-178.

[41] Y. Xu, "Pointnet_pointnet2_pytorch," https://github.com/yanx27/ Pointnet_Pointnet2_pytorch 2019.

[42] Q. Huang, W. Wang, and U. Neumann, "Recurrent slice networks for 3d segmentation of point clouds," in Proceedings of the IEEE Conference on Computer Vision and Pattern Recognition, 2018, pp. 2626-2635.

[43] L. Yi, H. Su, X. Guo, and L. J. Guibas, "Syncspeccnn: Synchronized spectral cnn for $3 \mathrm{~d}$ shape segmentation," in Proceedings of the IEEE Conference on Computer Vision and Pattern Recognition, 2017, pp. 2282-2290.

[44] Y. Wang, Y. Sun, Z. Liu, S. E. Sarma, M. M. Bronstein, and J. M. Solomon, "Dynamic graph cnn for learning on point clouds," Acm Transactions On Graphics (tog), vol. 38, no. 5, pp. 1-12, 2019.

[45] Y. Xu, T. Fan, M. Xu, L. Zeng, and Y. Qiao, "Spidercnn: Deep learning on point sets with parameterized convolutional filters," in Proceedings of the European Conference on Computer Vision (ECCV), 2018, pp. $87-102$.

[46] M. Tatarchenko, J. Park, V. Koltun, and Q.-Y. Zhou, "Tangent convolutions for dense prediction in 3d," in Proceedings of the IEEE Conference on Computer Vision and Pattern Recognition, 2018, pp. 3887-3896.

[47] P. Hermosilla, T. Ritschel, P.-P. Vázquez, À. Vinacua, and T. Ropinski, "Monte carlo convolution for learning on non-uniformly sampled point clouds," ACM Transactions on Graphics (TOG), vol. 37, no. 6, pp. $1-12,2018$.

[48] A. Jaegle, F. Gimeno, A. Brock, A. Zisserman, O. Vinyals, and J. Carreira, "Perceiver: General perception with iterative attention," 2021. 